\title{
Optic nerve head circulation in untreated ocular hypertension
}

Gilbert T Feke, Bernard Schwartz, Takenori Takamoto, Naoki Fujio, Suguru Konno, Douglas G Goger, Vinay Nangia

\begin{abstract}
Aims-The laser Doppler technique was used to compare the capillary blood speed measured at localised sites of the optic nerve head in stable, untreated ocular hypertensive patients with that measured in healthy normal subjects. The stereophotogrammetric technique was also used to measure the retinal nerve fibre layer thickness at the disc margin in the eyes of the patients.

Methods-Doppler broadening measurements were made at superior and inferior temporal disc sites in 18 eyes of 10 ocular hypertensive patients and in 12 eyes of seven age and sex-matched normal subjects.

Results-On average, Doppler broadening and, hence, capillary blood speed were significantly higher $(p=0.018)$ in the patients than in the normal subjects. The largest values of Doppler broadening in the patients were measured at sites adjacent to the thinnest retinal nerve fibre layer. Linear regression analysis showed a significant inverse relation $(p=0.0004)$ between Doppler broadening and nerve fibre layer thickness in left eyes, and a nearly significant relation $(p=0.06)$ in right eyes. At temporal sites of the optic nerve head there is a compensatory relation between a thinning nerve fibre layer and a locally increasing blood supply to the optic nerve head.
\end{abstract}

Conclusion-Together with previous observations of fluorescein filling defects in similar patients, these results indicate that there is spatial heterogeneity of blood flow in the optic nerve head in stable, untreated ocular hypertensive patients. (Br f Ophthalmol 1995; 79: 1088-1092)

The continuing recognition that the classic signs of open angle glaucoma may occur even at normal intraocular pressure levels, ${ }^{1}$ coupled with the fact that glaucomatous visual field loss occurs in only $30 \%$ of patients with elevated intraocular pressures even when followed for 20 years, ${ }^{23}$ has led investigators to examine mechanisms other than elevated intraocular pressure alone in the pathogenesis of glaucomatous damage. ${ }^{4}$ The role of the ocular circulation in the onset and progression of glaucoma had previously been studied using fluorescein angiography. ${ }^{5}$ There has been a resurgence of research in this field fuelled by the development of new quantitative, non-invasive techniques for the assessment of the ocular circulation. ${ }^{6}$ Thus, Wolf et $a l^{7}$ using a scanning laser technique, have recently reported that a pronounced circulatory deficit exists within the retinal vasculature in eyes of patients with chronic open angle glaucoma. This finding was seen in both treated and untreated patients. In addition, they found that the blood plasma viscosity was significantly elevated in the patients compared with that measured in healthy, non-glaucomatous subjects. Also, Hamard et l $^{8}$ used the laser Doppler technique to assess the microcirculation at localised sites of the optic nerve head in patients under treatment for primary open angle glaucoma and in untreated patients with normal pressure glaucoma. They found that in both patient groups the blood flow velocity was reduced compared with that measured in healthy subjects. In their accompanying haemorheological studies, they found that the red blood cells of the patients exhibited hyperaggregability, requiring abnormally high shear rates in order to dissociate.

In our study, we used the laser Doppler technique to compare the capillary blood speed measured at localised sites of the optic nerve head in stable, untreated ocular hypertensive patients with that measured in healthy normal subjects. We also used the stereophotogrammetric technique to measure the retinal nerve fibre layer thickness at the disc margin in the eyes of the patients, and examined the relation between the local circulatory measurements and the local nerve fibre layer thickness.

\section{Patients and methods}

Subjects for the study included 10 patients with untreated ocular hypertension (two males, eight females), ranging in age from 42 to 75 (mean 58.4) years, and seven normal subjects free of ocular or systemic diseases (two males, five females), ranging in age from 50 to 75 (mean 63.6) years. The procedures performed in the study followed the tenets of the Declaration of Helsinki, and were approved by the Schepens Eye Research Institute human studies committee. Written informed consent was obtained from each patient.

The patients had intraocular pressures greater than or equal to $21 \mathrm{~mm} \mathrm{Hg}$ and less than $35 \mathrm{~mm} \mathrm{Hg}$ by Goldmann applanation tonometry on two or more independent examinations. All patients had open angles on slit-lamp gonioscopy. One patient had increased pigmentation of the angles. Each patient had normal visual fields in both eyes as determined by automated perimetry with the 
Octopus PERIMETER, programs 31 or G1. The patients' optic discs had varying amounts of cupping and pallor, but each disc had an area of neural rim that was sufficiently large for laser Doppler measurements. There had been no changes in the clinical appearance of the disc, retinal nerve fibre layer, or visual fields over the previous several years. Each patient had been followed for at least 2 years before the study. The mean earlier follow up period was $7 \cdot 25$ years. The means of the intraocular pressures measured during the 2 year period before the study ranged from $18.2 \mathrm{~mm} \mathrm{Hg}$ to $24.5 \mathrm{~mm} \mathrm{Hg}$. None of the patients had any specific systemic diseases except for one who was being treated for hypothyroidism, and none was on systemic or topical medications such as corticosteroids, $\beta$ blockers, or calcium channel blockers that would affect the vascular system or the intraocular pressure. The patients had no previous ocular surgery, laser treatment, or other ocular disease. The normal subjects were volunteers with no history of ocular problems or of systemic hypertension, diabetes, stroke, or transient cerebral attack.

On the day of laser Doppler testing, intraocular pressure ranged from 18 to 25 (mean $22 \cdot 1) \mathrm{mm} \mathrm{Hg}$ among the patients, and from 14 to 20 (mean 16.9) $\mathrm{mm} \mathrm{Hg}$ among the normal subjects. The difference in intraocular pressure between the patients and the normal subjects was statistically significant $(p=0.0001)$. Following measurement of intraocular pressure, subjects' pupils were dilated with $1 \%$ tropicamide and $2.5 \%$ phenylephrine (neosynephrine). While the effect of topical application of these drugs on the optic nerve head circulation has not been measured, it has been shown that they do not produce any changes in retinal blood flow. ${ }^{9}$

The methodology of our application of the laser Doppler technique to the measurement of the speed of red blood cells flowing in the capillaries of the optic nerve head has been described in detail. ${ }^{10}$ When a region of optic nerve head tissue is illuminated by laser light (633 $\mathrm{nm}$ in our application), the frequency shift spectrum of the scattered light is Doppler broadened in direct proportion to the speed of the red blood cells flowing through the capillaries. Measurements of the Doppler broadening according to the algorithm we have described ${ }^{10}$ provide a reliable means by which to compare optic head blood speed from one site to another and from one subject to another.

Doppler broadening measurements were obtained from a superior temporal and an inferior temporal site on the optic nerve head in each eye studied. The sites were approximately $180 \mu \mathrm{m}$ in diameter, in regions free of surface vessels, and were generally located midway between the outer margin of the pallor ${ }^{11}$ and the margin on the nerve head. Five separate Doppler broadening measurements each of 5.12 seconds in duration were obtained at each of the sites. The Doppler broadening value at each site (DB) was computed as the average of the five measurements. The mean Doppler broadening in each eye studied
(DBeye) was computed by averaging the DB value obtained at the superior site with that obtained at the inferior site.

Both eyes were studied in eight of the patients; only the left eye was studied in the other two patients. Among the normals, both eyes were studied in five subjects; only the right eye was studied in the other two subjects. Media opacities interfered with the laser Doppler measurements in the eyes that were not studied. The mean Doppler broadening for each subject studied (DBsubject) was computed by averaging the DBeye value obtained in the right eye with that obtained in the left eye. In the subjects in which only one eye was studied, DBsubject was the same as DBeye.

The retinal nerve fibre layer thickness (NFLT) at the disc margin was measured in the 10 ocular hypertensive patients from simultaneous stereophotographs ( $3 \times$ magnification) of the optic disc taken with a Donaldson fundus camera. ${ }^{12}$ The photogrammetric technique used for these measurements has been described in detail. ${ }^{13}$ NFLT at the disc margin is defined as the depth difference between the internal limiting membrane of the retina and the top surface of the retinal pigment epithelium. The measurements were made at $5^{\circ}$ intervals around the disc margin in each eye studied. The results were expressed as the ratio of the measured thickness to the vertical disc radius (VDR), an approach previously found to minimise eye to eye magnification differences on the measurements. ${ }^{13}$ Measurements were made in triplicate in both eyes in seven of the patients. Single measurements were made in the remaining eyes studied. The mean coefficient of variation of the three measurements at each site was 5\% (SD 3\%).

The laser Doppler measurements and NFLT/VDR measurements were masked in relation to each other. Since a laser Doppler measurement involves approximately a $20^{\circ}$ sector of the disc, averages of five separate $5^{\circ}$ NFLT measurements centred around the points adjacent to the centre points of the laser Doppler measurements were computed and used in the analysis in which Doppler broadening and NFLT were compared.

The statistical significance of differences in Doppler broadening and intraocular pressure between the patients and normal subjects, and between right and left eyes in both groups, was determined using two tailed, two sample, $t$ tests. Linear regression analysis was used to examine the relations between Doppler broadening and age and intraocular pressure in both groups, and between Doppler broadening and NFLT/VDR in the patients. Spearman correlation analysis was used to examine the relations between the Doppler broadening measured at the two temporal disc sites in each eye, and between the Doppler broadening measured at similar sites in right and left eyes. The same analysis was also used to study the site to site relations between the local NFLT/VDR measurements in the patients. A $p$ value of 0.05 or less was considered statistically significant. 


\section{Results}

The DB value at each of the measurement sites in the ocular hypertensive patients and the normal subjects are shown in Tables 1 and 2 . The mean coefficient of variation of the five measurements at each of the sites was $9 \%$ (SD $4 \%$ ). In the ocular hypertensive patients, the mean Doppler broadening in each eye (DBeye) was $937(148) \mathrm{Hz}$ in left eyes $(n=10)$ and $907(95) \mathrm{Hz}$ in right eyes $(n=8)$. In the normal subjects, DBeye was 845 (111) $\mathrm{Hz}$ in left eyes $(n=5)$ and $808(157) \mathrm{Hz}$ in right eyes $(n=7)$. There were no significant differences in DBeye between left and right eyes in the patients or in the normal subjects.

Figure 1 shows the mean Doppler broadening for each subject (DBsubject) measured in the ocular hypertensive patients and in the normal subjects. In the patients, the group average DBsubject $(n=10)$ was $935(96) \mathrm{Hz}$; in the normal subjects, the group average DBsubject $(n=7)$ was $797(119) \mathrm{Hz}$. The $17 \%$ difference was statistically significant $(p=0 \cdot 018)$.

Linear regression analysis showed no significant correlations between Doppler broadening and intraocular pressure measured just before laser Doppler testing in the patients or in the normal subjects, or between Doppler broadening and mean intraocular pressure over the previous 2 years in the patients. Linear regression analysis did show an inverse trend $(r=-0.68, \mathrm{p}=0.096)$ in the relation between Doppler broadening and age in the normal

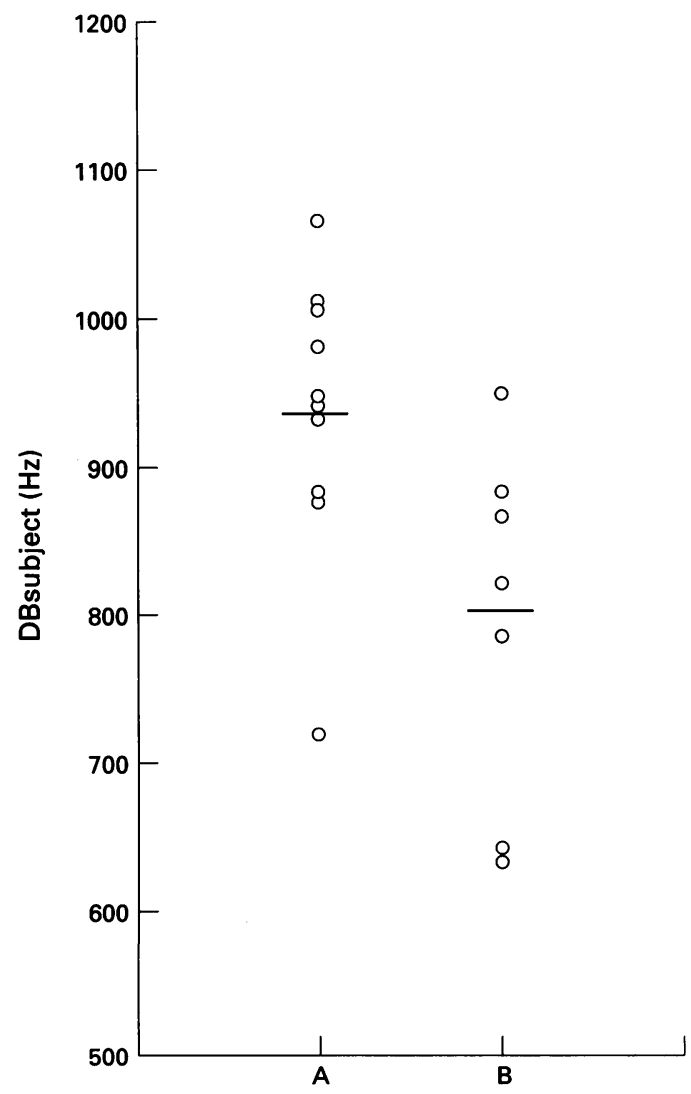

Figure 1 The mean Doppler broadening for each subject (DBsubject) measured in untreated ocular hypertensive patients $(A)$ and in normal subjects $(B)$. Doppler

broadening is directly proportional to the speed of red blood cells flowing through the capillaries of the optic nerve head. Lines indicate mean value for each group.
Table 1 Doppler broadening (DB) measured at temporal sites of the optic nerve head in ocular hypertensive patients

\begin{tabular}{lccccc}
\hline & \multicolumn{2}{l}{ Left eye $(\mathrm{DB}(\mathrm{Hz}))$} & & \multicolumn{2}{c}{ Right eye $(\mathrm{DB}(\mathrm{Hz}))$} \\
\cline { 2 - 3 } Subject & Superior & Inferior & & Superior & Inferior \\
\hline 1 & 651 & 723 & & 949 & 1203 \\
2 & 530 & 847 & & 813 & 686 \\
3 & 826 & 852 & & 1129 & 693 \\
4 & 1225 & 771 & & 805 & 982 \\
5 & 1131 & 912 & & 1091 & 880 \\
6 & 1010 & 1193 & & 886 & 829 \\
7 & 938 & 1037 & & 1032 & 750 \\
8 & 975 & 965 & & 642 & 1138 \\
9 & 1028 & 1102 & & - & - \\
10 & 830 & 1186 & & - & - \\
\hline
\end{tabular}

Table 2 Doppler broadening (DB) measured at temporal sites of the optic nerve head in normal subjects

\begin{tabular}{lcclll}
\hline & \multicolumn{2}{c}{ Left eye $(D B(\mathrm{~Hz}))$} & & \multicolumn{2}{c}{ Right eye $(\mathrm{DB}(\mathrm{Hz}))$} \\
\cline { 2 - 3 } Subject & Superior & Inferior & & Superior & Inferior \\
\hline 1 & 850 & 815 & & 1177 & 953 \\
2 & 955 & 894 & & 711 & 967 \\
3 & 1077 & 876 & & 657 & 850 \\
4 & 679 & 705 & & 960 & 937 \\
5 & 652 & 948 & & 717 & 818 \\
6 & - & - & & 688 & 601 \\
7 & - & - & & 704 & 565 \\
\hline
\end{tabular}

subjects that was not as apparent $(r=-0 \cdot 19$, $\mathrm{p}=0.595)$ in the patients. In both patients and normal subjects, Spearman correlation analysis showed no significant relations between the Doppler broadening measured at superior versus inferior sites in left or right eyes, or between the Doppler broadening measured in left versus right eyes at superior or inferior sites.

Spearman correlation analysis was also used to examine the site to site relations between NFLT/VDR measured at the disc margin adjacent to the laser Doppler measurement sites in the patients. There were no significant correlations between NFLT/VDR measured at superior versus inferior sites in left or right eyes, or between NFLT/VDR measured in left versus right eyes at superior sites. However, there was a significant correlation $(p=0.04)$ in NFLT/VDR measured in left versus right eyes at inferior sites. Therefore, the relation between Doppler broadening and NFLT/VDR was analysed separately for left and right eyes.

Figure 2 shows the Doppler broadening measured at each site plotted as a function of NFLT/VDR adjacent to the site for left (A) and right (B) eyes. Linear regression analysis showed a significant inverse relation $(r=$ $-0.72, \mathrm{p}=0.0004)$ in left eyes and an inverse trend $(r=-0.48, p=0.06)$ in right eyes. The largest values of Doppler broadening were measured at sites adjacent to the thinnest retinal nerve fibre layer.

\section{Discussion}

Our finding of elevated blood speeds in the capillaries of the optic nerve head in the ocular hypertensive patients might seem surprising in light of the results reported by Hamard et $a l^{8}$ in studies on primary open angle glaucoma patients and on normal pressure glaucoma patients. While optic nerve head blood speeds were found to be abnormally decreased in those patient groups, our results show an 

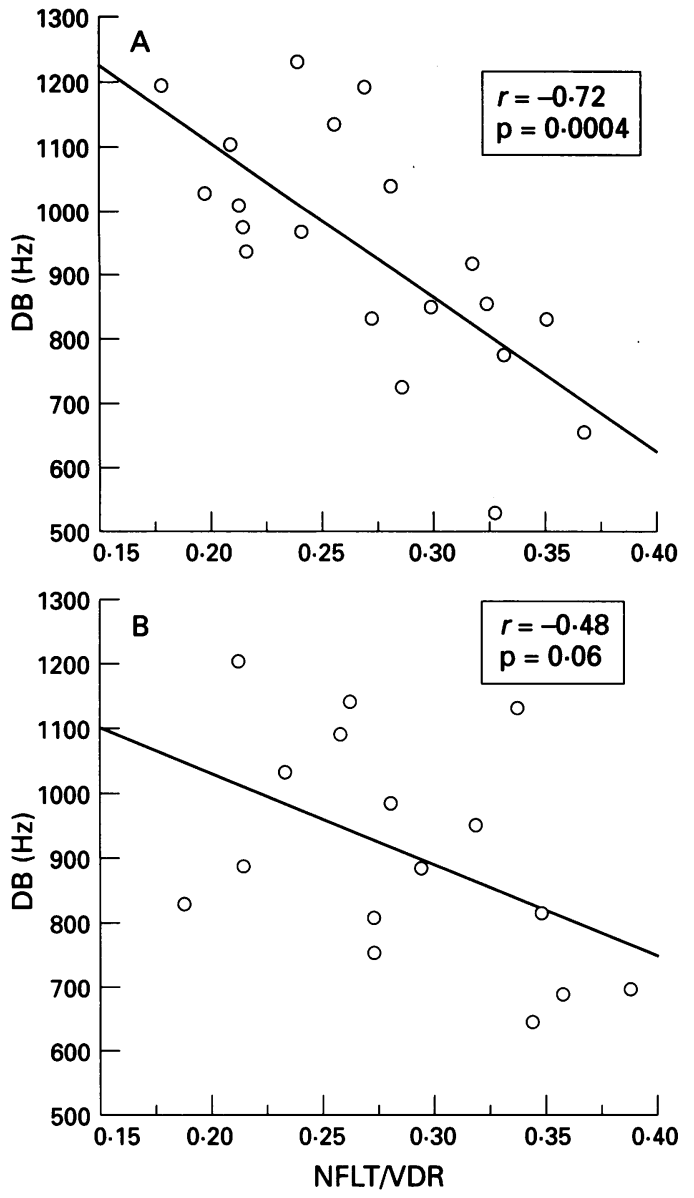

Figure 2 Doppler broadening (DB) measured at superior and inferior temporal disc sites in untreated ocular hypertensive patients plotted as a function of the retinal nerve fibre layer thickness divided by the vertical disc radius (NFLT/VDR) measured at the disc margin adjacent to the laser Doppler measurement site. Results are shown separately for left eyes ( $A$ ) (20 sites) and right eyes (B) (16 sites). Lines represent the linear regression fits to the data.

abnormal increase in optic nerve head blood speed in clinically stable, untreated ocular hypertensive patients.

The only previous studies of the optic nerve head circulation in ocular hypertensive patients have been qualitative - utilising fluorescein angiography to locate regions of hypofluorescence, indicating circulatory deficits, on the optic disc. Nanba and Schwartz ${ }^{14}$ examined primary open angle glaucoma patients and ocular hypertensive patients for these filling defects of the optic disc and for the retinal nerve fibre layer defects. They found that while filling defects could be observed in all 31 glaucoma patients studied, they were present in only 14 out of the 43 ocular hypertensive patients studied. Furthermore, Fishbein and Schwartz ${ }^{15}$ had previously shown that the filling defects of the optic disc in glaucoma patients tended to cluster at the inferior and superior poles, with most sites located slightly nasally. In our ocular hypertensive patients, only six of the 36 laser Doppler measurements sites were located near the inferior or superior poles, with the remainder located in the temporal quadrant. Our finding of elevated blood speeds at localised disc sites is therefore not in direct conflict with the fluorescein studies, but instead serves to emphasise the spatial heterogeneity of optic nerve head microcirculatory changes. Finally, it should be pointed out that the observations of the fluorescein studies were not analysed to determine whether there was any evidence of areas of increased perfusion at localised disc sites.

In a previous study of open angle glaucoma patients with advanced visual field loss, Lee and Schwartz ${ }^{16}$ found that significant differences were obtained when eyes with and without cilioretinal arteries were compared for retention of central visual field and visual acuity. They hypothesised that the presence of a temporal cilioretinal artery might provide an enhanced blood supply to the temporal rim of the optic disc which would aid in the retention of the central field and acuity. In our ocular hypertensive patients, eight of the 36 laser Doppler measurement sites were located near cilioretinal arteries. On average, the Doppler broadening measured at these sites was $9 \%$ higher than at the other sites, but the difference was not statistically significant.

It is interesting to note that while Wolf $e^{~} a l^{7}$ and Hamard et $\mathrm{al}^{8}$ found abnormal haemorheological characteristics in patients with open angle glaucoma or normal pressure glaucoma, Garcia-Salinas et al ${ }^{17}$ found haemorheological differences in patients with ocular hypertension compared with patients with open angle glaucoma. Specifically, they found that blood viscosity was significantly higher in open angle glaucoma patients than in ocular hypertensive patients or control subjects, but that blood haematocrit was significantly lower in the ocular hypertensive patients than in glaucoma patients or control subjects. They speculated that the low haematocrit observed in the patients with ocular hypertension may act to normalise the overall blood viscosity and in some measure protect these patients from glaucomatous damage.

We had previously shown ${ }^{10}$ that optic nerve head blood speed in normal subjects decreases with age in the range 31 to 76 years. Our finding of a similar trend in this study is consistent with that result. Interestingly, we did not observe such an apparent decrease of blood speed with age in the ocular hypertensive patients.

Our results do show that the elevated optic nerve head capillary blood speeds occur specifically in regions adjacent to the thinnest retinal nerve fibre layer. The inverse linear relation between Doppler broadening and NFLT/VDR was statistically significant in left eyes $(n=20)$ and nearly statistically significant in right eyes $(n=16)$. It is likely that the results would have been statistically significant in right eyes as well if the number of measurement sites in right eyes were the same as in left eyes.

Our finding suggests that either (1) a thinner nerve fibre layer alters the depth of the optic nerve tissue that is sampled by the laser Doppler measuring beam, ${ }^{18}$ perhaps allowing the beam to sample deeper vessels with higher speeds, or (2) there is a compensatory relation between a thinning nerve fibre layer and a locally increasing blood supply to the optic nerve head. The first alternative is not likely. 
Takamoto and Schwartz ${ }^{13}$ have shown that patients with primary open angle glaucoma have a thinner nerve fibre layer than ocular hypertensive patients. Yet, Hamard et al using a laser Doppler technique similar to ours have reported that optic nerve head capillary blood speeds are abnormally low in both primary open angle glaucoma and normal pressure glaucoma patients. Their results are in agreement with our unpublished findings in such patients.

Thus, our results suggest that there is a compensatory relation between a thinning nerve fibre layer and a locally increasing blood supply. The increased blood supply may serve to maintain the viability of the nerve fibre layer, and thus forestall the onset of visual field loss in these stable ocular hypertensive patients.

The inference from our capillary blood speed measurements that there are localised increases in the blood supply to the optic nerve head in ocular hypertensive patients depends upon assumptions involving the capillaries themselves. Increased blood speed translates into increased blood flow only if the capillaries are not constricted and only if the number of capillaries per tissue volume is not decreased. Experimental studies conducted by Quigley et $a l^{19}$ on glaucomatous optic nerves showed that the number of capillaries per unit volume of tissue did not change with neural loss and that capillary diameters in glaucomatous eyes were the same as in contralateral normal eyes. We assume, therefore, that since capillary diameters and number of capillaries per tissue volume are not different from normal in glaucomatous eyes, they are also not different from normal in ocular hypertensive eyes.

An interesting parallel exists between our findings and the temporal modulation perimetry results recently reported by Casson and Johnson. ${ }^{20}$ In measurements of the sensitivity to sinusoidal flicker in ocular hypertensive patients, early glaucoma patients, and normal subjects, they found that the glaucoma patients showed reduced sensitivity compared with normal, while the ocular hypertensive patients showed, on average, a slightly increased sensitivity compared with normal, reflecting the fact that both abnormally high and abnormally low sensitivities were measured in the visual fields of the patients with ocular hypertension. When the patients with ocular hypertension were separated into two groups, those that subsequently progressed to visual field loss and those that remained stable, it was found that the abnormally high sensitivity to flicker occurred mainly in the group that remained stable.

In summary, we are led to speculate that the eventual onset of visual field loss in ocular hypertensive patients may coincide with either the attainment of a minimum nerve fibre layer thickness for which circulatory compensation is not possible, or the failure of a mechanism that facilitates the compensatory circulatory increase. An investigation into the nature of such a mechanism could be beneficial in the understanding and treatment of glaucomatous visual field loss.

1 Grosskreutz C, Netland PA. Low-tension glaucoma. Int Ophthalmol Clin 1994; 34: 173-85.

2 Hovding G, Aasved H. Prognostic factors in the development of manifest open angle glaucoma. Acta Ophthalmol 1986; 64: 601-8.

3 Lundberg L, Wettrell K, Linner E. Ocular hypertension: a prospective twenty-year follow-up study. Acta Ophthalmol 1987; 65: 705-8.

4 Drance SM. Bowman lecture. Glaucoma - changing concepts. Eye 1992; 6: 337-45.

5 Schwartz B, Kern J. Age, increased ocular and blood pressures, and retinal and disc fluorescein angiogram. pressures, and retinal and disc fluo

6 Schwartz B, ed. Ocular blood flow in the progression and therapy of glaucoma. Surv Ophthalmol 1994; 38 (suppl): $\mathrm{S} 1-\mathrm{S} 208$

7 Wolf S, Arend O, Sponsel WE, Schulte K, Cantor LB, Reim M. Retinal hemodynamics using scanning laser ophthalmoscopy and hemorheology in chronic open-angle glaucoma. Ophthalmology 1993; 100: 1561-6.

8 Hamard P, Hamard H, Dufaux J, Quesnot S. Optic nerve head blood flow using a laser Doppler velocimeter and haemorheology in primary open angle glaucoma and normal pressure glaucoma. $\mathrm{Br} \mathcal{F}$ Ophthalmol 1994; 78: normal

9 Robinson F, Petrig BL, Sinclair SH, Riva CE, Grunwald JE. Does topical phenylephrine, tropicamide, or proparacaine affect macular blood flow? Ophthalmology 1985; 92: $1130-2$

10 Rizzo JF, Feke GT, Goger DG, Ogasawara H, Weiter JJ. Optic nerve head blood speed as a function of age in normal human subjects. Invest Ophthalmol Vis Sci 1991; 32: 3263-72.

11 Schwartz B. Cupping and pallor of the optic disc. Arch Ophthalmol 1973; 89: 272-7.

12 Donaldson D, Prescott R, Kennedy S. Simultaneous stereoscopic fundus camera incorporating a single optical axis. scopic fundus camera incorporating a single

13 Takamoto T, Schwarz B. Photogrammetric measurement of nerve fiber layer thickness. Ophthalmology 1989; 96: $1315-9$

14 Nanba K, Schwartz B. Nerve fiber layer and optic disc fluorescein defects in glaucoma and ocular hypertension. Ophthalmology 1988; 95: 1227-33.

15 Fishbein SL, Schwartz B. Optic disc in glaucoma: topography and extent of fluorescein filling defects. Arch Ophthalmol 1977; 95: 1975-9.

16 Lee SS, Schwartz B. Role of the temporal cilioretinal artery in retaining central visual field in open-angle glaucoma. in retaining central visual field in

17 Garcia-Salinas P, Trope GE, Glynn M. Blood viscosity in ocular hypertension. Can $\mathcal{F}$ Ophthalmol 1988; 23: 305-7.

18 Koelle JS, Riva CE, Petrig BL, Cranstoun SD. Depth of tissue sampling in the optic nerve head using laser flowmetry. Lasers in Medical Science 1993; 8: 49-54.

19 Quigley HA, Hohman RM, Addicks EM, Green WR. Blood vessels of the glaucomatous optic disc in experimental promate and human eyes. Invest Ophthalmol Vis Sci 1984; 25: $918-31$.

20 Casson EJ, Johnson CA. Temporal modulation perimetry in glaucoma and ocular hypertension. In: Millis RP, ed. New York: Kugler Publications, 1993: 443-50. 